\title{
A Modified Trimmed Median Filter Technique for Noise Removal in an Image
}

\author{
Bhaskara Rao Jana, Beatrice Seventline.J
}

\begin{abstract}
A modified trimmed filtration that is actually median when it comes to recovering the original images that are dishonored by the salt \& pepper noise is actually presented here.The refined the pixels tend to be checked for high or least pixel values for example, 0 or 255 in this suggested method. Then this expedient pixel is recognized as the noisy one otherwise maybe not a noisy one in the event that processed pixel is actually 0 or 255. A two-dimensional $3 * 3$ window can end up being selected with this specific noisy pixel as center component and being a running pixel. In this $3 * 3$ window, then this algorithm can replace the value of the loud pixel as mean of these 9 elements in the $3 * 3$ screen if all 9 aspects in the window are values like 0's or 255 's. Then this modified trimmed median filtration technique can be applied if most of the 9 elements tend to be not 0's or 255's. This formula can eliminate the 0's and present that is 255 's the $3 * 3$ window and change the loud pixel value as average of mean and median of this remaining pixel in $3 * 3$ screen. Simulation results imply that the proposed modified trimmed filter that is actually median can be works really in the event that picture can end up being influenced by salt and pepper sound.The outcome obtained from the recommended modified trimmed filter is compared with the AWMF, DBUTVF, and MDBUTMF. This proposed MTMF filtering technique is actually analyzed on various images for different quality testing parameters like PSNR, MSE.
\end{abstract}

Keywords - high density noise, salt and pepper noise, MeanMedian filter, TMF.

\section{INTRODUCTION}

In the image processing, the more research happens to be accomplished on image noise removal in recent years. The digital images are generally degraded by many types of noises such as salt and pepper noise.

The images that can frequently degraded by the salt and pepper noise because of broadcast that is bad or ambiguity in the acquisition product or defective mind location channels in equipment. The pepper and salt sound requires high and reduced values associated with the pictures [8], [9]. The pre-processing procedure in image control is actually blocking that is noise. The filter systems that tend to be created should remove noise without removing the given info of a picture. Adaptive weighted filtration that is mean that good in reducing/ removing the salt-and-pepper noise but do never to eliminate high-density sound. A Noise adaptive fuzzy switching filtration this is certainly median can adaptively change the window size. Special filter systems like CBAFSMF [6] adaptive vector median filter [10] were suggested. Decision Based trimmed Algorithms [1], [2], [3] happened to be suggested by some researchers.

Revised Manuscript Received on April 12, 2019.

BhaskaraRao Jana, Department ofECE, Anil Neerukonda Institute of Technology\& Sciences, Visakhapatnam, A.P, India. (E-mail: janabhaskar@gmail.com)

Beatrice Seventline.J,Department ofECE, GIT, GITAM deemed to be University, Visakhapatnam, A.P, India. (E-mail: samsandra2003@yahoo.com)
A MDBMF was recommended [1], [2]. This algorithm changed with unsymmetrical average instead of conventional median. At greater noise densities [4], [5] the formula lead in fading. The MDBMF algorithm ended up being refined with MDBUTMF [1], [2]. The formula also exhibits fading[7] at larger sound densities. The MDBUTMPF algorithm replaced the pixel this is certainly degraded Unsymmetrical trimmed midpoint. The formula presented here dealt with in literatures operates for equal probability pepper-and-salt sound. The Paper addresses the overall performance of the proposed formula both for equivalent and noise this is certainly unequal. Section 2 deals with picture restoration filter systems. Section 3 provides the proposed algorithm part 4 gives knowledge associated with proposed algorithm part 5 shows the representation effects of proposed filtration with present filter systems. Section 6 provides in conclusion.

\section{IMAGE RESTORATION FILTERS}

The image restoration can be used to restore the images which tend to be degraded by noise using filtering that is different enhancement strategies. Digital picture repair is really a field of technology that scientific studies practices used to recuperate an world that is original degraded observations[5]. The picture destruction and renovation that is subsequent depicted like in Figure 1. In this thesis, nonetheless, only part this is certainly noise of destruction is actually worked with, which is found in Figure 2.

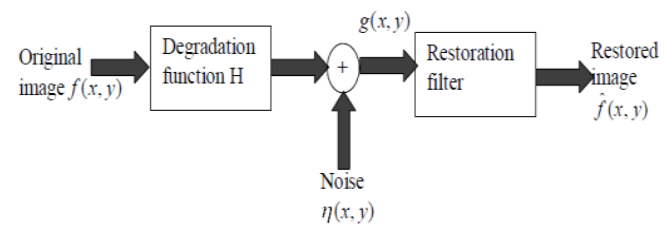

Fig. 1.Image degradation and restoration model

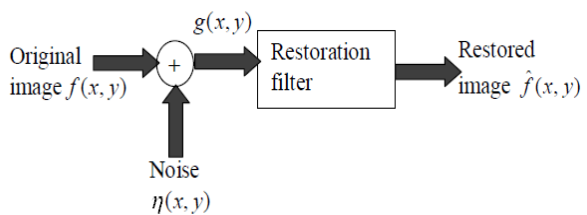

Fig. 2.Noise removal process model

\section{THE PROPOSED MTMFALGORITHM}

The proposed algorithm for the noise removal i.e., Modified Trimmed Median Filter (MTMF) is elucidated in

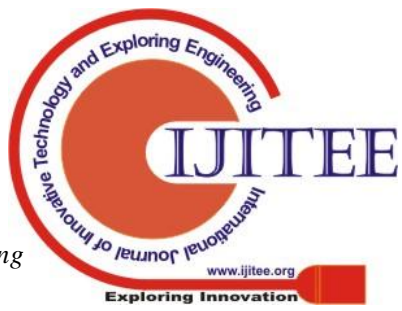


this part.Here, a $3 * 3$ window is selected from the image and in the selected $3 \times 3$ window, the processing pixel is inspected whether the processing pixel is degraded by the noise or not. It has to find from the value of the pixel, when pixel value of the processing pixel is in between the utmost and least gray level values of this image, then it termed as a non-noisy/ noise free pixel otherwise noisy one. If the processing pixel is detected as a noise free pixel then this kept unchanged. When the processing pixel value is the utmost and least gray level values of this image, then it termed as a noisy pixel. When it is the noisy pixel, then this can be replaced with the MTMF calculated for this window. Here, the noisy image is represented with $\mathrm{N}(\mathrm{i} . \mathrm{j})$ and the restored image is represented with $\mathrm{K}(\mathrm{i}, \mathrm{j})$

The various steps involved in the MTMF are described below.

Step 1: Read the input image which is noisy $\mathrm{N}(\mathrm{i}, \mathrm{j})$

Step 2: Select a processing pixel $\mathrm{N}_{\mathrm{ij}}$, if this processing pixel $0<\mathrm{N}_{\mathrm{ij}}<255$, then it's taken as a non-noisy pixel and $\mathrm{N}_{\mathrm{ij}}$ is kept same, Else step 3.

Step 3: With this processing pixel, $\mathrm{N}_{\mathrm{ij}}$ as center element and as processing pixel, Select a 2 D $3 * 3$ window

Step 4: $\mathrm{N}_{\mathrm{ij}}$ is a degraded pixel, when $\mathrm{N}_{\mathrm{ij}}$ value is 0 or 255 then then the following two instants are possible. These two cases are depends on the all the elements present in the selected window.

Case a): The selected $3 * 3$ window contains 0 's and 255 's only, then $\mathrm{N}_{\mathrm{ij}}$ can be replaced with the mean of the all the elements in the $3 * 3$ window.

Case b): The selected $3 * 3$ window contains not only 0 's and 255's and other values too, then eliminate the 255's and 0 's present in the window and replace the $\mathrm{N}_{\mathrm{ij}}$ with the average of mean and median value calculated for the outstanding elements present in the window.

Step 5: recur steps 1 to 3 until and unless all the pixels that are present in the image are covered further to get restored image $\mathrm{K}(\mathrm{i}, \mathrm{j})$

\section{WORKING OF MTMF ALGORITHM}

The input present pixel $\mathrm{N}_{\mathrm{ij}}$ is actually checked for the lowest or the highest pixel values i.e., 0 or 255 on all the pixels present in the original image. The big matrix relates to this image and values enclosed within a rectangle are to function as handling window this is certainly current. The processed pixel represented as encircled element. Fig 3 gives the flowchart of MTMF algorithm is illustrated in fig 3.

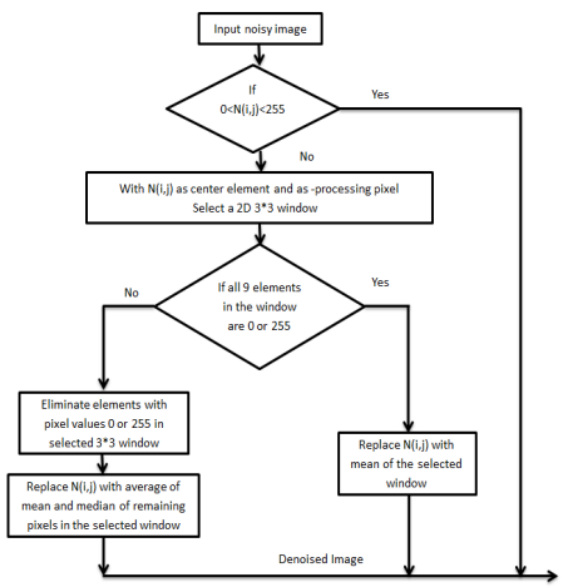

Fig. 3.The Flowchart of the MTMF algorithm (Proposed)

Case 1: Take any pixel from the image as the selected pixel is noisy one (i.e., the pixel value is either 0 or 255) and also some of the four neighbors of the selected pixel are also the noisy ones.

Let the processing pixel value is 255 (represented in circle) and check for the neighbors of the processing pixel and arrange them in increasing order.

Un-sorted Array: 155, 255, 255, 0, 255, 123, 255, 164, 255

Sorted Array: 0, 123, 155, 164, 255, 255, 255, 255, 255

The processing pixel value in the matrix looks as a noisy one and this can be replaced by the modified trimmed mean i.e., mean median average of $(123,155,164)$ is 151 which changes the degraded pixel value as illustrated in Figure 4.

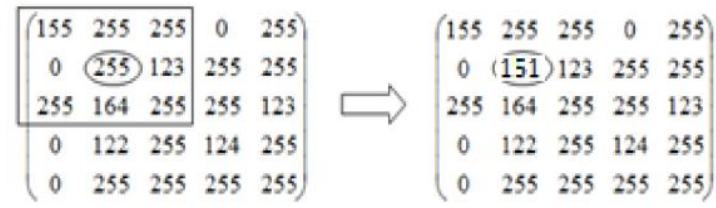

Part of the Original Image.Part of the Restored Image.

Fig. 4.Explanation of case 1

Case 2: If the selected pixel is actually a noisy one and most of the elements within the selected window are tend to be noisy. Here, the problem with the present pixel is acts as a nosy but a non-noisy one. For this type of issues, searches for the four nearest neighbor pixels of the pixel this is certainly processed all are 255), Here as shown in the fig 5, all the neighbors of the selected pixel are 0's and 255's only i.e., noisy. The pixel that is actually dishonored is replaced by the proposed, that will be given as $\left(255^{* 5}\right) / 9=170$ as revealed in Figure 4.

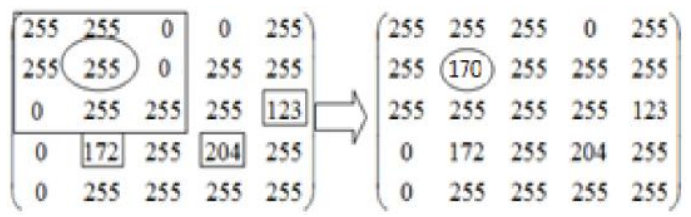

Part of the Original Image.Part of the Restored Image.

Fig.5Explanation of case 2 .

Published By: 
Case 3: When the pixel is noisy; most of the four nearest neighbors tend to be the noisy ones. The refined pixel is loud that is 0 , then it check for the four neighbors regardingthe prepared pixel (that are $0,255,255,255$ ) in this case. It had been unearthed that all the four next-door neighbors tend to be noisy; Hence get suggest of the four neighbors which will be computed as the average of the four neighbors i.e., 191. The present pixel is actually loud, the four next-door neighbors of the present pixel is additionally loud and therefore replace the pixel that is loud mean for the 4 neighbors (which can be 191) as explained in Figure 5.

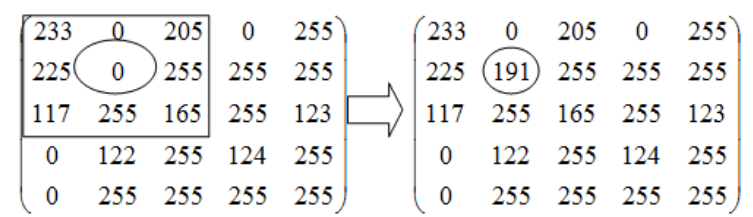

Part of the Original Image.Part of the Restored Image.

Fig. 6.Explanation of case 3.

Case 4: If the processed pixel in the selected window is a non-noisy pixel the refined pixel is taken as 119 and the pixel value is lies in between the upmost and least pixel values i.e., 0 and 255 . So, this processed pixel is referred to as a non-processed and loud pixel is actually unchanged as illustrated in Figure 6. This MTMF algorithm is described in the shape of flowchart as shown in Figure 7.

$\left(\begin{array}{ccc|cc}0 & 0 & 255 & 0 & 255 \\ 104 & 119 & 255 & 255 & 255 \\ 0 & 103 & 255 & 255 & 123 \\ 0 & 122 & 255 & 124 & 255 \\ 0 & 255 & 255 & 255 & 255\end{array}\right) \square\left(\begin{array}{ccc|cc}0 & 0 & 255 & 0 & 255 \\ 104 & 119 & 255 & 255 & 255 \\ 0 & 103 & 255 & 255 & 123 \\ 0 & 122 & 255 & 124 & 255 \\ 0 & 255 & 255 & 255 & 255\end{array}\right)$

Part of the Original Image.Part of the Restored Image.

Fig. 7.Explanation of case 5.

\section{SIMULATION RESULTS}

The quantitative performance of this proposed MTMF algorithm is assessed by considering PSNR and MSE.

PSNR and MSE are provided in the Equation 1, 2 respectively.

$$
\begin{aligned}
& P S N R=10 \cdot \log _{10}\left(\frac{M A X_{I}^{2}}{M S E}\right) \\
& M S E=\frac{1}{m n} \sum_{i=0}^{m-1} \sum_{j=0}^{n-1}[I(i, j)-K(i, j)]^{2}
\end{aligned}
$$

Where we relate to original non noisy image, $\mathrm{N}$ provides image that isdegraded is actually denotes recovered image, $\mathrm{m} \times \mathrm{n}$ may be the measurements of prepared image.

The filters such as AWMF, DBUTVF [2], and MDBUTMF were utilized when it comes to assessment. Thetable 1, 2, gives the comparison of numerous formulas for PSNR and MSE on standard image like Cameraman, which was degraded by the Salt and pepper noise.
TABLE I. COMPARISON OF THE VARIOUS

EXIXTING ALGORITHMS FOR PSNR ON CAMERAMAN IMAGE DEGRADED BY SALT AND PEPPER NOISE.

\begin{tabular}{|c|c|c|c|c|}
\hline $\begin{array}{c}\text { ND } \\
\text { in } \\
\text { \% }\end{array}$ & AWMF & DBUTVF[2] & MDBUTMF & Proposed \\
\hline $\mathbf{1 0}$ & 32.3449 & 33.6949 & 35.4310 & 35.6688 \\
\hline $\mathbf{2 0}$ & 30.6532 & 30.2353 & 31.9828 & 31.9138 \\
\hline $\mathbf{3 0}$ & 28.9350 & 28.1888 & 29.3196 & 29.5950 \\
\hline $\mathbf{4 0}$ & 27.9045 & 25.9284 & 28.0828 & 28.0241 \\
\hline $\mathbf{5 0}$ & 26.4356 & 24.4475 & 26.0357 & 26.3195 \\
\hline $\mathbf{6 0}$ & 25.4247 & 22.4904 & 24.0993 & 24.9381 \\
\hline $\mathbf{7 0}$ & 23.9492 & 20.7468 & 21.1907 & 23.6858 \\
\hline $\mathbf{8 0}$ & 22.4947 & 18.9591 & 17.8266 & 22.1959 \\
\hline $\mathbf{9 0}$ & 20.3158 & 17.5238 & 13.9893 & 20.3324 \\
\hline
\end{tabular}

TABLE II.COMPARISON OF THE VARIOUS EXIXTINGALGORITHMS FOR MSE ON CAMERAMAN IMAGE DEGRADED BY SALT AND PEPPER NOISE.

\begin{tabular}{|c|c|c|c|c|}
\hline $\begin{array}{c}\text { ND } \\
\text { in } \\
\text { \% }\end{array}$ & AWMF & DBUTVF[2] & MDBUTMF & Proposed \\
\hline $\mathbf{1 0}$ & 37.8959 & 27.7711 & 18.6202 & 17.6278 \\
\hline $\mathbf{2 0}$ & 55.9449 & 61.5964 & 41.1908 & 41.8507 \\
\hline $\mathbf{3 0}$ & 83.0956 & 98.6739 & 76.0538 & 71.3802 \\
\hline $\mathbf{4 0}$ & 105.3479 & 166.0489 & 101.1123 & 102.4871 \\
\hline $\mathbf{5 0}$ & 147.7475 & 233.5243 & 161.9982 & 151.7490 \\
\hline $\mathbf{6 0}$ & 186.4685 & 366.4699 & 253.0198 & 208.5802 \\
\hline $\mathbf{7 0}$ & 261.9167 & 547.5210 & 494.3264 & 278.2913 \\
\hline $\mathbf{8 0}$ & 366.1087 & 826.3590 & $1.0726 \mathrm{e}+03$ & 392.1867 \\
\hline $\mathbf{9 0}$ & 604.6503 & $1.1500 \mathrm{e}+03$ & $2.5951 \mathrm{e}+03$ & 602.3413 \\
\hline
\end{tabular}

The graphical illustrations of the performance of various algorithms such as AWMF, DBUTVF [2], and MDBUTMFover the proposed MTMF algorithm on the standard image cameraman image for PSNR and MSE respectively.

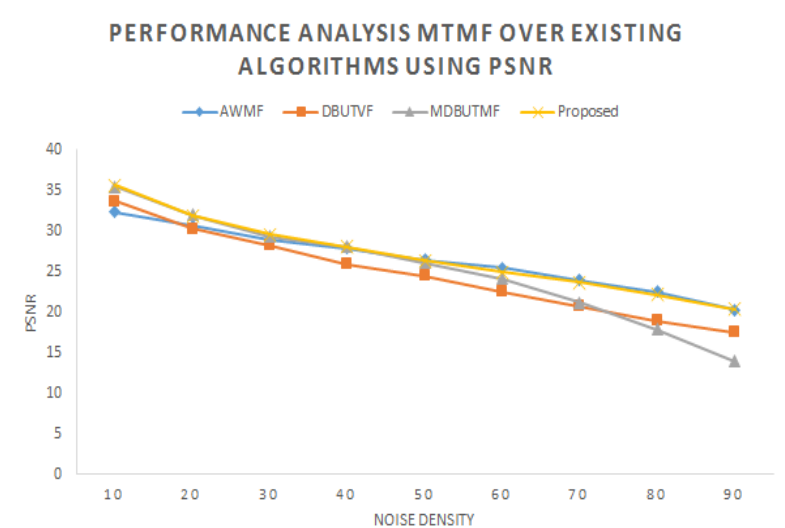

Fig. 8.Performance of the PSNR for MTMF algorithm over existing algorithms. 
PERFORMANCE ANALYSIS MTMF OVER EXISTING ALGORITHMS USING MSE

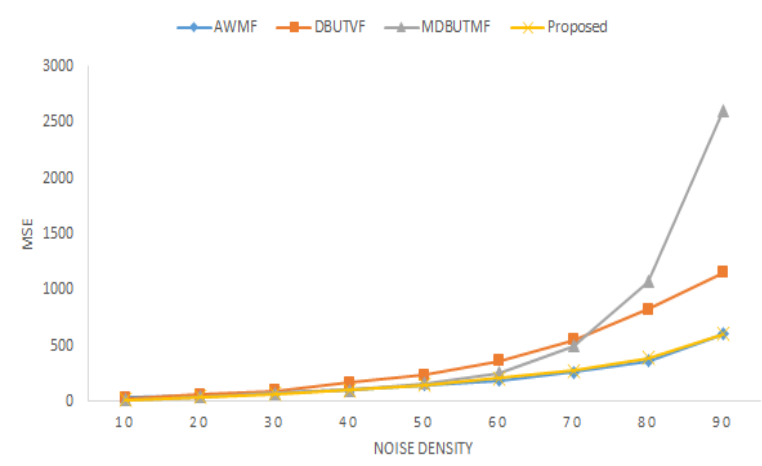

Fig. 9.Performance of the MSE for MTMF algorithm over existing algorithms.

Thequalitative performance the various existing algorithms on a standard image such as cameraman image which is degraded by salt and pepper noise for various noise densities $70 \%$ to $90 \%$ is shown in Figure 10 .

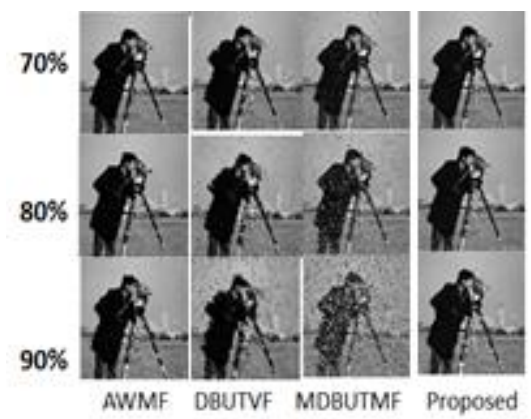

Fig. 10.Performance analysis of the various algorithms for cameraman image degraded by $70 \%, 80 \%, 90 \%$ (shown in row 1 to 3 ) with salt and pepper noise. The results of different algorithms are illustrated in column.

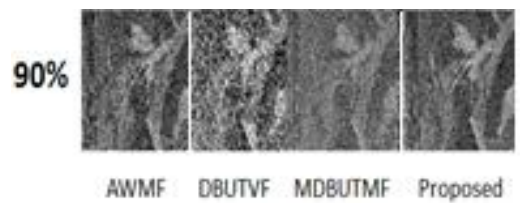

Fig. 11.Performance analysis of various algorithms on standard image such asLena which isdegraded by $90 \%$ salt and pepper noise. The results of different algorithm illustrated in column.

Figure 11 provides the qualitative results of numerous formulas degraded by pepper-and-salt noise at $90 \%$. It had been found through the tables that the DBTVMF fairs less at method and sound this is certainly high when compared to MTMF. The MTMF shows good by exhibiting a higher PSNR with lower MSE for high noise densities. This means that that the proposed algorithm reveals much better causes sound removal. A quality of PSNR suggests that the recommended algorithm reveals noise this is certainly good characteristics, enhanced top quality of an image after sound reduction and excellent information preserving capabilities respectively. The quantitative performance of the proposed algorithm was located great at larger noise densities and in addition discovered to demonstrate good result this is certainly visual various images.

\section{CONCLUSION}

This modified trimmed median filter (MTMF) is suggested here for the restoration of the images which are degraded by salt \& pepper noise. The proposed work operates in the pixel by pixel based. The suggested MTMF shows really great by exhibiting a higher PSNR with reduced MSE for different noise densities. This indicates that the proposed formula reveals greater results in sound removal. A quality value of PSNR indicates that the recommended algorithm reveals noise that's good characteristics, enhanced high quality of an image after noise removing and exceptional information preserving features respectively. This formula is actually not at all hard and exhibits extremely great results with regards to both quantitative and qualitative actions through the effects obtained by contrasting this suggested algorithm with existing methods. Hence this algorithm would work for recovering the original images which are degraded by the salt \&pepper noise.

\section{REFERENCES}

1. Vasanth, K., T. G. Manjunath, and S. Nirmal Raj. "A Decision based Unsymmetrical Trimmed Modified Winsorized Mean Filter for the Removal of High Density Salt and Pepper Noise in Images and Videos." Procedia Computer Science 54 (2015): 595-604

2. K.Vasanth, V.Jawahar senthilkumar,Rajesh.V “A Decision Based Unsymmetrical Trimmed Variants for the Removal of High Density Salt and Pepper Noise" International Journal of Computer Applications (0975 - 8887) Volume 42- No.15, March 2012.

3. Akkoul, Smaïl, et al. "A new adaptive switching median filter." IEEE Signal Processing Letters 17.6 (2010): 587-590.

4. Irum, Isma, et al. "A nonlinear hybrid filter for salt \& pepper noise removal from color images." Journal of applied research and technology 13.1 (2015): 79-85.

5. Beagum, S. Samsad, and S. Sheeja. "A Novel Restoration Technique for the Elimination of Salt and Pepper Noise using 8-Neighbors based Median Filter." Advances in Computational Sciences and Technology 10.9 (2017): 28512874.

6. Toh, Kenny Kal Vin, and Nor Ashidi Mat Isa. "Cluster-based adaptive fuzzy switching median filter for universal impulse noise reduction." IEEE Transactions on Consumer Electronics56.4 (2010).

7. Roy, Amarjit, et al. "Combination of adaptive vector median filter and weighted mean filter for removal of high-density impulse noise from colour images." IET Image Processing11.6 (2017): 352-361.

8. Lal, Shyam, and Mahesh Chandra. "Efficient Algorithm for Enhancement of Images Degraded by Salt \& Pepper Noise." WSEAS Transactions on Signal Processing 8.3 (2012): 135144.

9. Babu, K. Ratna, et al. "Image Denoising in the Presense of High Level Salt and Pepper Noise using Modified Median Filter." IJCST 2.1 (2011): 180-3.

10. Shrestha, Suman. "Image denoising using new adaptive based median filters." arXiv preprint arXiv:1410.2175 (2014). 